\title{
Control Systems of the Large Millimeter Telescope
}

\author{
W. Gawronski ${ }^{1}$ and K. Souccar ${ }^{2}$
}

\begin{abstract}
This article presents the analysis results (in terms of settling time, bandwidth, and servo error in wind disturbances) of four control systems designed for the Large Millimeter Telescope (LMT). The first system, called the PP system, consists of the proportional-and-integral (PI) controllers in the rate and position loops and is widely used in the antenna and radio telescope industry. The analysis shows that the PP control system performance is remarkably good when compared to similar control systems applied to typical antennas. This performance is achieved because the LMT structure is exceptionally rigid, but the performance does not meet the stringent pointing requirements of the LMT. The second system, called the PL system, consists of the PI controller in the rate loop and the linear-quadraticGaussian (LQG) controller in the position loop. It is implemented at the NASA Deep Space Network antennas, and its pointing precision is twice as good as the PP control system. The third system, called the LP system, consists of the LQG controller in the rate loop and the proportional-integral-derivative (PID) controller in the position loop. It has not been implemented yet at known antennas or radio telescopes, but the analysis shows that its pointing accuracy is ten times better than the PP control system. The fourth system, called the LL system, consists of the LQG controller in the rate loop and in the position loop. It is the best of the four (its precision is 250 times better than the PP system); thus, it is worth further investigation and verification of implementation challenges for telescopes with high pointing requirements.
\end{abstract}

\section{Introduction}

The Large Millimeter Telescope (LMT) Project is a joint effort of the University of Massachusetts at Amherst and the Instituto Nacional de Astrofísica, Óptica, y Electrónica (INAOE) in Mexico. The LMT is a 50-m-diameter millimeter-wave radio telescope (see Fig. 1) designed for principal operation at wavelengths between $1 \mathrm{~mm}$ and $4 \mathrm{~mm}$. The telescope is being built atop Sierra Negra (4640 m), a volcanic peak in the state of Puebla, Mexico. Site construction and fabrication of most of the major antenna parts is under way, with telescope construction expected to be complete in 2005 .

\footnotetext{
${ }^{1}$ Communications Ground Systems Sections.

${ }^{2}$ LMT Project, Astronomy Department, University of Massachusetts, Amherst.

The research described in this publication was carried out by the Jet Propulsion Laboratory, California Institute of Technology, and was partially funded by the National Science Foundation and the Advance Research Project Agency, Sensor Technology Office, issued by DARPA/CMO.
} 


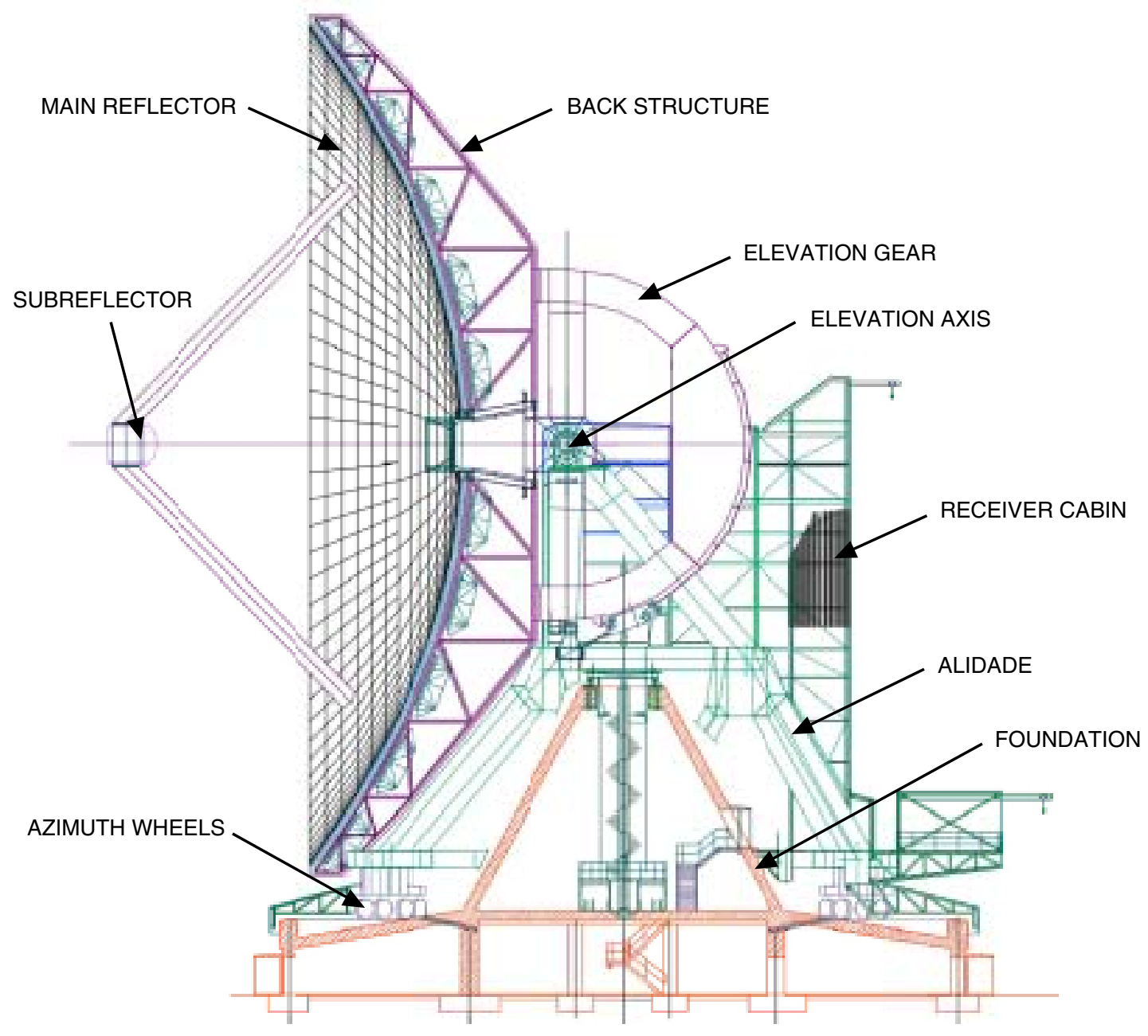

Fig. 1. A drawing of the Large Millimeter Telescope. The whole structure rotates with respect to the vertical axis (azimuth) on the azimuth wheels, and the dish rotates with respect to the horizontal (elevation) axis.

The LMT will be a significant step forward in antenna design since, in order to reach its pointing and surface accuracy specifications, it must outperform every other telescope in its frequency range. The largest existing telescope with surface error superior to the LMT is the 15-m James Clerk Maxwell Telescope, located at the summit of Mauna Kea (4092 m) in Hawaii, and there is no telescope of any size that reaches the LMT pointing requirements. The antenna designer expects that this system will point the telescope to its specified accuracy of 1 arcsec under conditions of low winds and stable temperatures. However, under the maximum operating wind conditions, the pointing will degrade to a few arcseconds (rms). The pointing challenges and their solutions are discussed in [1] and [8].

It is known that the linear-quadratic-Gaussian (LQG) controllers guarantee wide bandwidth and good wind-disturbance rejection properties [2]; thus, they are used for antennas with stringent pointing requirements in the presence of wind disturbances. For antennas and radio telescopes, the LQG control systems can be implemented in two different ways: at the telescope rate loop or at the position loop. This article analyzes and compares the performances of the LQG controllers at these two locations. The analysis is augmented with the performance of the proportional-and-integral (PI) controller. The latter analysis is necessary not only for comparison purposes (the PI controller is a standard antenna industry feature), but also because the implementation of the LQG controller requires preliminary installation of PI controllers in both rate and position loops. The implementation of the LQG algorithm needs an 
accurate telescope model, and it can be obtained from the field test of the telescope. Thus, a telescope will be operational before the LQG controller implementation.

Why do we need an accurate rate-loop model? It can be explained as follows. The performance of a controller improves with the gain increase. However, high gains excite structural vibrations. For a PI controller, structural vibrations cannot easily be controlled since the structural deformations are not directly measured by encoders. But an LQG controller uses a Kalman filter to estimate telescope vibrations, overcoming the difficulty of direct measurement. The Kalman filter consists of a telescope analytical model that needs to be accurate to produce an accurate estimate of the telescope structural dynamics.

The control system of the LMT consists of rate and position loops, as shown in Fig. 2. Four control systems will be analyzed. They have the following structure:

(1) PP control system, with a PI controller in the position loop and a PI controller in the rate loop

(2) PL control system, where the PI controller is in the rate loop and the LQG controller is in the position loop

(3) LP control system, where the LQG controller is in the rate loop and the PID (proportionalintegral-derivative) controller is in the position loop

(4) LL control system, where the LQG controller is in the rate loop and the LQG controller is in the position loop

The configurations of the control systems are presented in Table 1. The PP control system is a typical telescope control system configuration. The PL case is the configuration of the NASA Deep Space Network (DSN) antenna control system, and it was implemented at the 34-meter antennas in Goldstone, California. The LQG controller has been considered by MAN Technologie $[1,8]$. The LP and LL control systems have not been implemented yet.

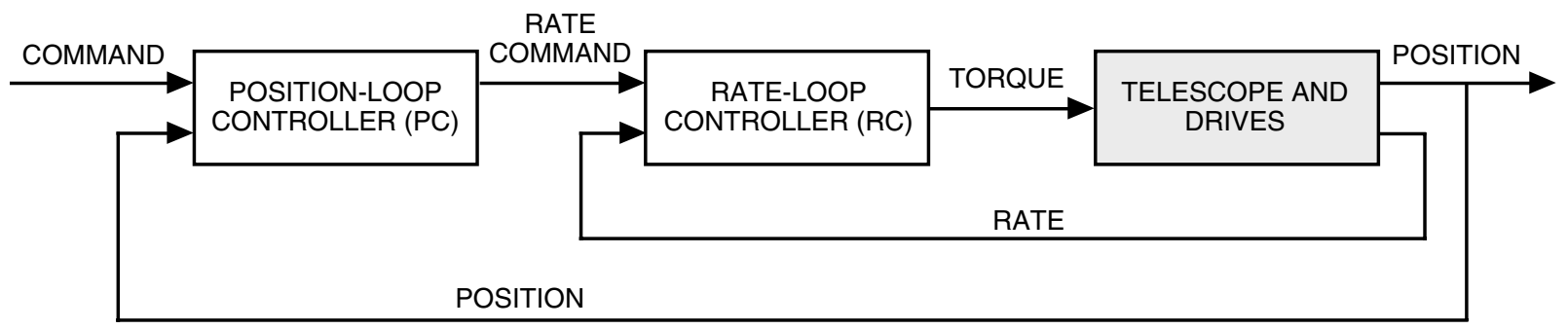

Fig. 2. Four control systems of the LMT: (1) the PP control system, where $R C=P I$ and $P C=P I,(2)$ the $P L$ control system, where $R C=P I$ and $P C=L Q G$, (3) the $L P$ control system, where $R C=L Q G$ and $P C=P I D$, and (4) the LL control system, where $R C=L Q G$ and $P C=L Q G$.

Table 1. Configurations of the control systems of the Large Millimeter Telescope.

\begin{tabular}{ccc}
\hline $\begin{array}{c}\text { LMT control } \\
\text { system }\end{array}$ & $\begin{array}{c}\text { Rate-loop } \\
\text { controller }\end{array}$ & $\begin{array}{c}\text { Position-loop } \\
\text { controller }\end{array}$ \\
\hline PP & PI & PI \\
PL & PI & LQG \\
LP & LQG & PID \\
LL & LQG & LQG \\
\hline
\end{tabular}


This article presents the performance analysis (in terms of bandwidth, step responses, and winddisturbance rejection properties) of the four control systems as applied to the LMT. This analysis will help to evaluate and select the control system not only for the LMT, but also for other antennas and radio telescopes of a similar design.

\section{The PP Control System}

The PP control system consists of a PI controller in the position loop and a PI controller in the rate loop. Its Simulink model is shown in Fig. 3(a) and the rate-loop subsystem in Fig. 3(b). The controller is shown in Fig. 3(c), with $k_{f}=0$. In this design, the position-loop PI controller is complemented with the feedforward (FF) loop to improve the tracking properties, especially at high rates, and with a command preprocessor (CPP) to avoid large overshoots during target acquisition and limit cycling during slewing.

\section{A. The Rate-Loop Model}

The rate-loop model is shown in Fig. 3(b). It consists of the finite-element model (FEM) of the telescope structure (labeled "Discrete Time FEM"), which includes the drives and the azimuth (AZ) and elevation (EL) rate-loop controllers. It is a discrete-time (digital) control system with a 0.001-s sampling time. The proportional-and-integral gains of the azimuth controller are 300; for the elevation controller, the proportional gain is also 300 , and the integral gain is 400 . The bandwidth of the rate-loop transfer function is $1.0 \mathrm{~Hz}$, both in azimuth and in elevation.

\section{B. Command Preprocessor}

Before the position loop is presented, we consider the rate and acceleration limits imposed at the drives. The acceleration limits prevent motors from overheating (the motor current is proportional to telescope acceleration). During tracking, the telescope motion is within the rate and acceleration limits. However, during slewing, the large position-offset commands exceed the acceleration limit or both the acceleration and rate limits. When limits are exceeded, the telescope dynamics are no longer linear, and the telescope becomes unstable, which is observed in the form of limit cycling (periodic motion of constant magnitude and of low frequency). Since the limit cycling is caused by commands that exceed the acceleration and rate limits, one easily can avoid the instability by properly shaping commands such that the limits are not exceeded.

The command preprocessor modifies the telescope commands such that they remain unaltered if they do not exceed the rate and acceleration limits, and it processes the command to the maximum acceleration and rate limits if the limits are exceeded by the command. The block diagram of the CPP is shown in Fig. 4. The CPP algorithm represents an integrator, rate and acceleration limits, a variable-gain controller, and a feedforward gain. Its input is a command $r$, and its output is the modified command $r_{f}$.

The variable gain $k$ depends on the CPP tracking error $e$ :

$$
k(e)=k_{o}+k_{v} e^{-\beta|e|}
$$

where $e=r-r_{f}$. For the LMT, we selected the following parameters: $k_{o}=0.3, k_{v}=1.0$, and $\beta=20$ for both azimuth and elevation. The plot of the gain $k$ versus the error $e$ is shown in Fig. 5. For more on the CPP, see [3].

Figure 6 shows how the CPP transforms a 10-deg step command for the LMT. The transformed step command shows the initial rise at the maximal acceleration, followed by maximal rate slope, and deceleration slowdown (which is smaller than the maximal deceleration in order to avoid excessive telescope shaking). By processing the commands, the CPP allows for smooth telescope responses to step offsets and eliminates telescope limit cycling during slewing (see Section II.D on the position-loop analysis). 


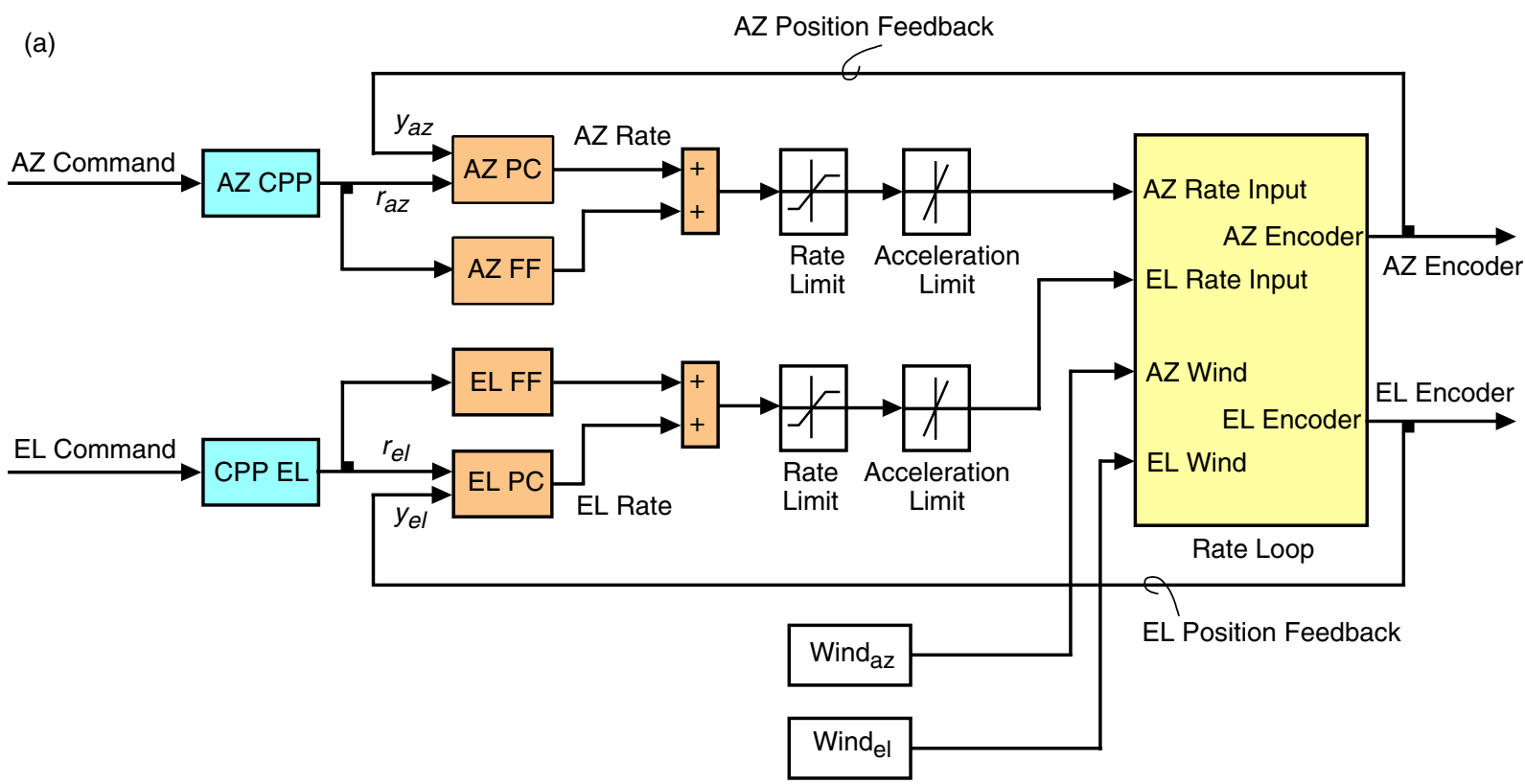

(b)

AZ Rate Feedback

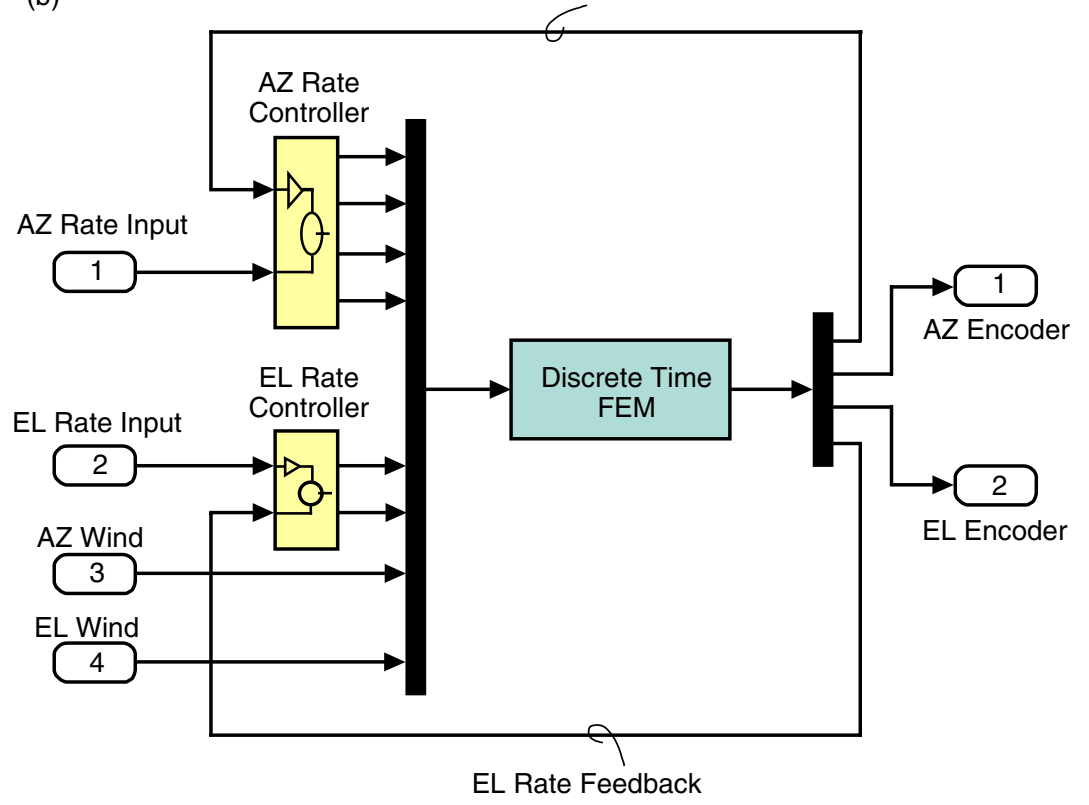

Fig. 3. The Simulink model of (a) the position loop, (b) the rate loop system, and (c) the controller (for $k_{f}=0$ it is a PI controller; for $k_{f} \neq 0$ it is an LQG controller). 


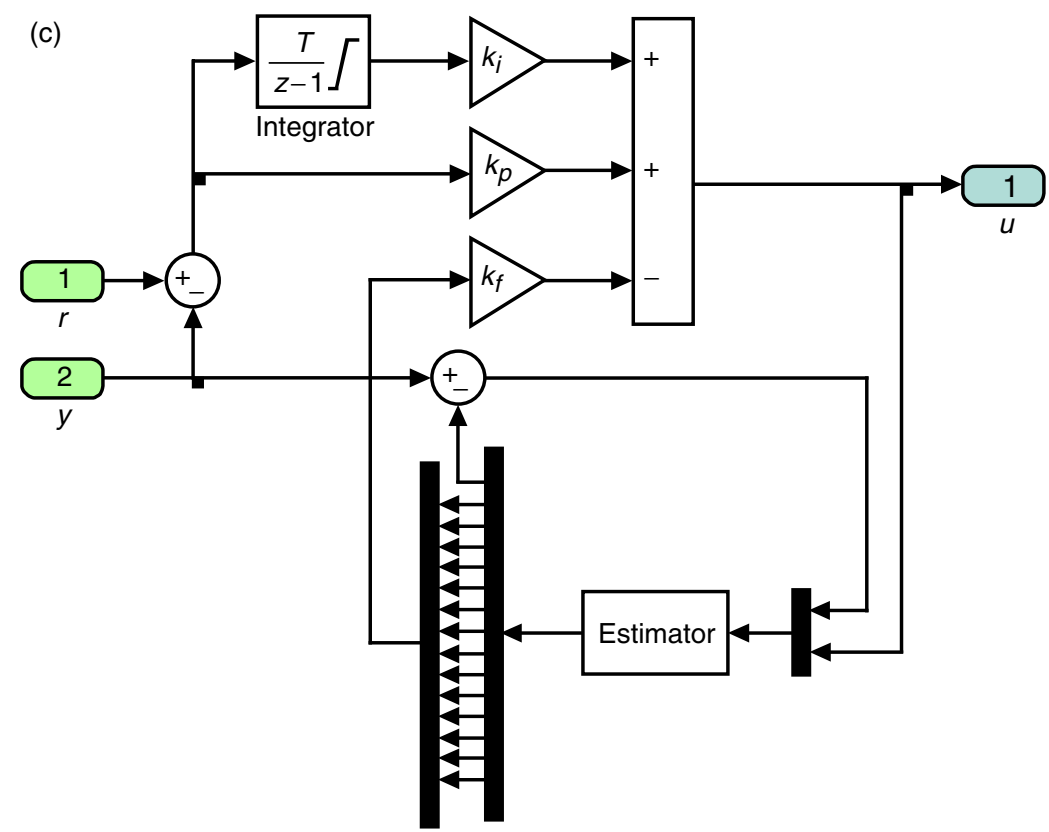

Fig. 3 (contd).

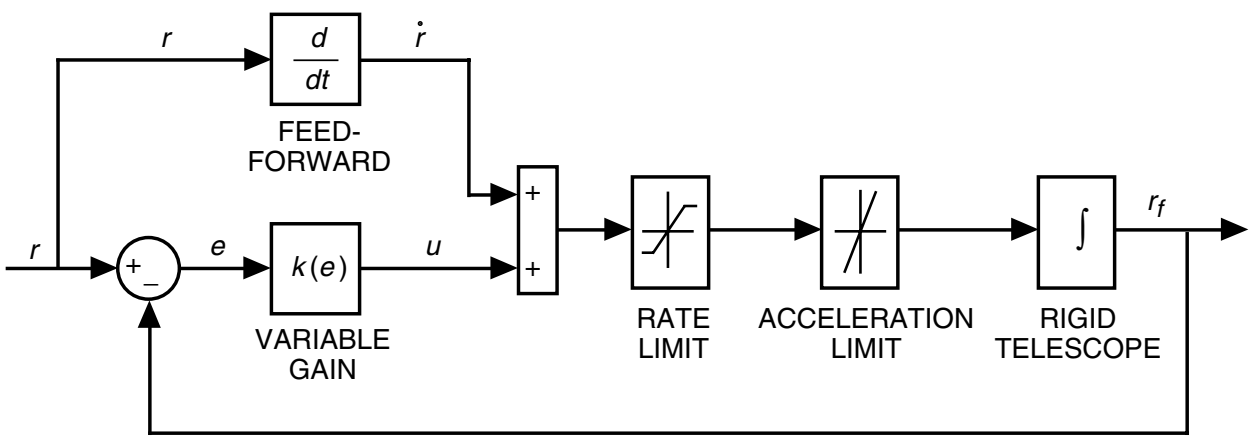

Fig. 4. The CPP block diagram.

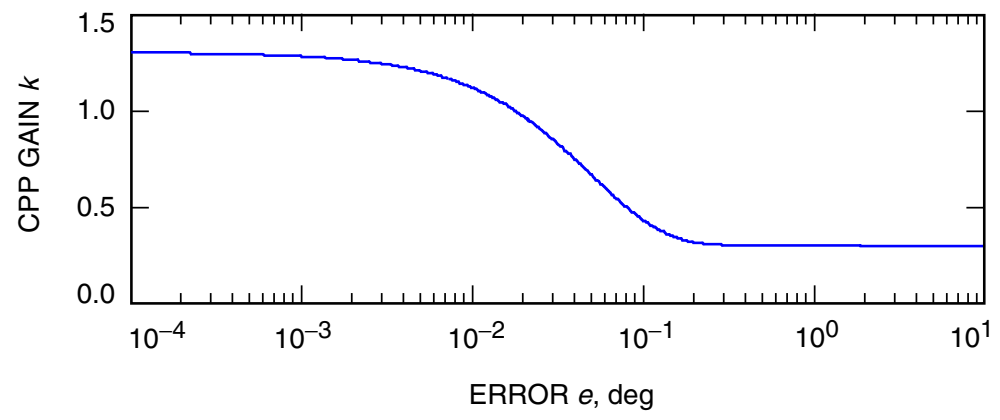

Fig. 5. CPP gain $k$ versus CPP error $e$. 


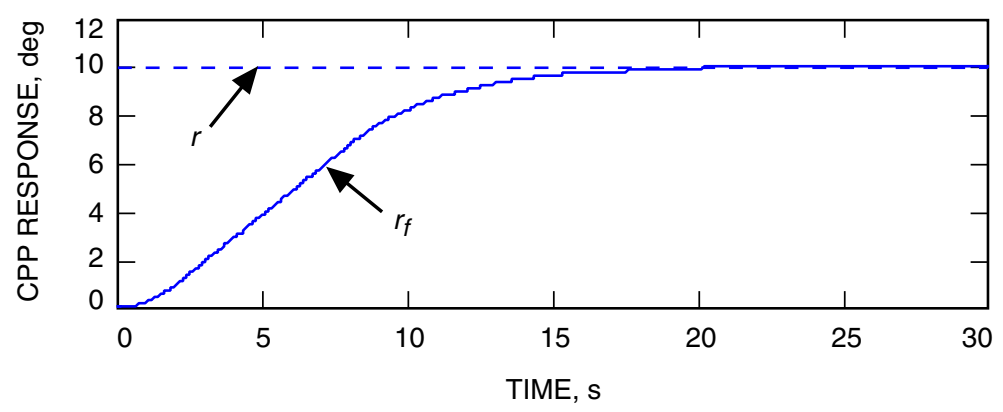

Fig. 6. CPP response $r_{f}$ to 10-deg step command $r$.

\section{Feedforward Loop}

The feedforward loop is added to improve tracking accuracy, especially at high rates. The feedforward loop differentiates the command and forwards it to the rate-loop input [see Fig. 3(a)]. The derivative is the inversion of the rate-loop transfer function. In this way, we obtain the open-loop transfer function from the command to the encoder, approximately equal to 1 . Indeed, the magnitude of the rate-loop transfer function $G_{r}$ is shown in Fig. 7. It can be approximated (up to $1 \mathrm{~Hz}$ ) with an integrator $\left(G_{\text {rapprox }}=1 / s\right.$ ), which is shown in the same figure (short-dashed line). The feedforward transfer function is a derivative $\left(G_{f f}(s)=s\right)$ shown in Fig. 7 (long-dashed line), so that the overall open-loop transfer function is a series connection of the feedforward and the rate loop $G_{o}(s)=G_{r}(s) G_{f f}(s)$, which is approximately equal to 1 up to a frequency of $1 \mathrm{~Hz}$. In this way, the transfer function of the system is equal to 1 (up to $1 \mathrm{~Hz}$ ) without applying the position feedback. The position feedback is added to compensate disturbances and system imperfections.

\section{Position Loop}

The position-loop model is shown in Fig. 3. It consists of the rate-loop model, PI and feedforward controllers in azimuth and elevation, command preprocessors in azimuth and elevation, and rate and acceleration limiters in azimuth and elevation. The telescope rate limit is $1.0 \mathrm{deg} / \mathrm{s}$, and the acceleration limit is $0.5 \mathrm{deg} / \mathrm{s}^{2}$, both in azimuth and elevation. The PI controller gains were selected to minimize settling time and servo error in wind gusts. They also guarantee zero steady-state error for constant rate tracking. The proportional gain is 3.0, and the integral gain is 1.0.

The position-loop transfer functions for azimuth and elevation are shown in Fig. 8. It follows from this figure that the azimuth bandwidth is $1.2 \mathrm{~Hz}$, while the elevation bandwidth is $1.8 \mathrm{~Hz}$ (the bandwidth is the frequency for which the magnitude of the transfer function falls below level 0.7). Note that the positionloop bandwidth is higher than the rate-loop bandwidth, both in azimuth and elevation. Note also that the elevation-axis bandwidth is higher than the azimuth-axis bandwidth, mainly because the elevation transfer function has only a few resonances and the resonances are well damped. Azimuth bandwidth is lower since the controller gains will be low in order to prevent excitation of multiple resonances.

In order to evaluate settling time, we simulated the step responses for small (0.01-deg) and large (3.0-deg) steps. The azimuth step responses are shown in Fig. 9 for the telescope with and without a CPP. From the plots, one can see that there is no overshoot when a CPP was implemented, and that the settling time is $3.0 \mathrm{~s}$ (small steps) in azimuth and elevation. Without a CPP, we observe overshoots for small steps and a settling time of $4.5 \mathrm{~s}$ in azimuth and elevation. For larger steps (e.g., when slewing), the telescope becomes unstable, showing limit cycling (see Fig. 9).

The wind-gust time history was obtained from the wind spectrum (see [4]). The plot of the servo errors in azimuth and elevation is shown in Fig. 10 (in black). 


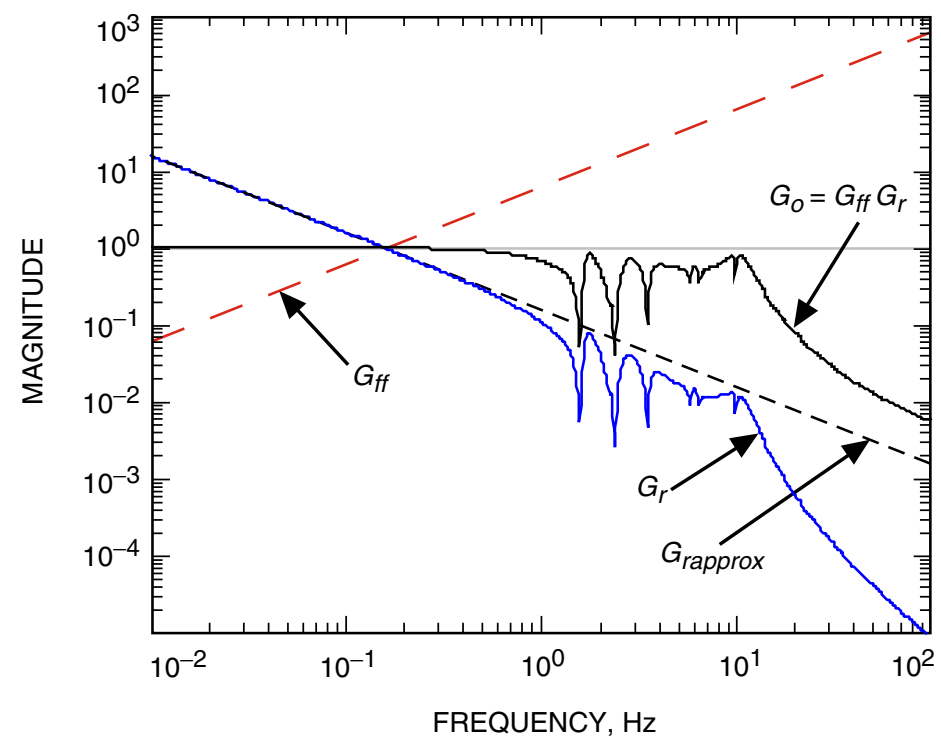

Fig. 7. The feedforward action illustrated by the magnitudes of the transfer functions $\left(G_{r}\right.$ is the rate-loop transfer function, $G_{\text {rapprox }}$ is the rate-loop transfer function approximation, $G_{f f}$ is the feedforward loop transfer function, and $G_{O}=G_{f f} G_{r}$ is the transfer function of the series connection of feedforward and rate loops).
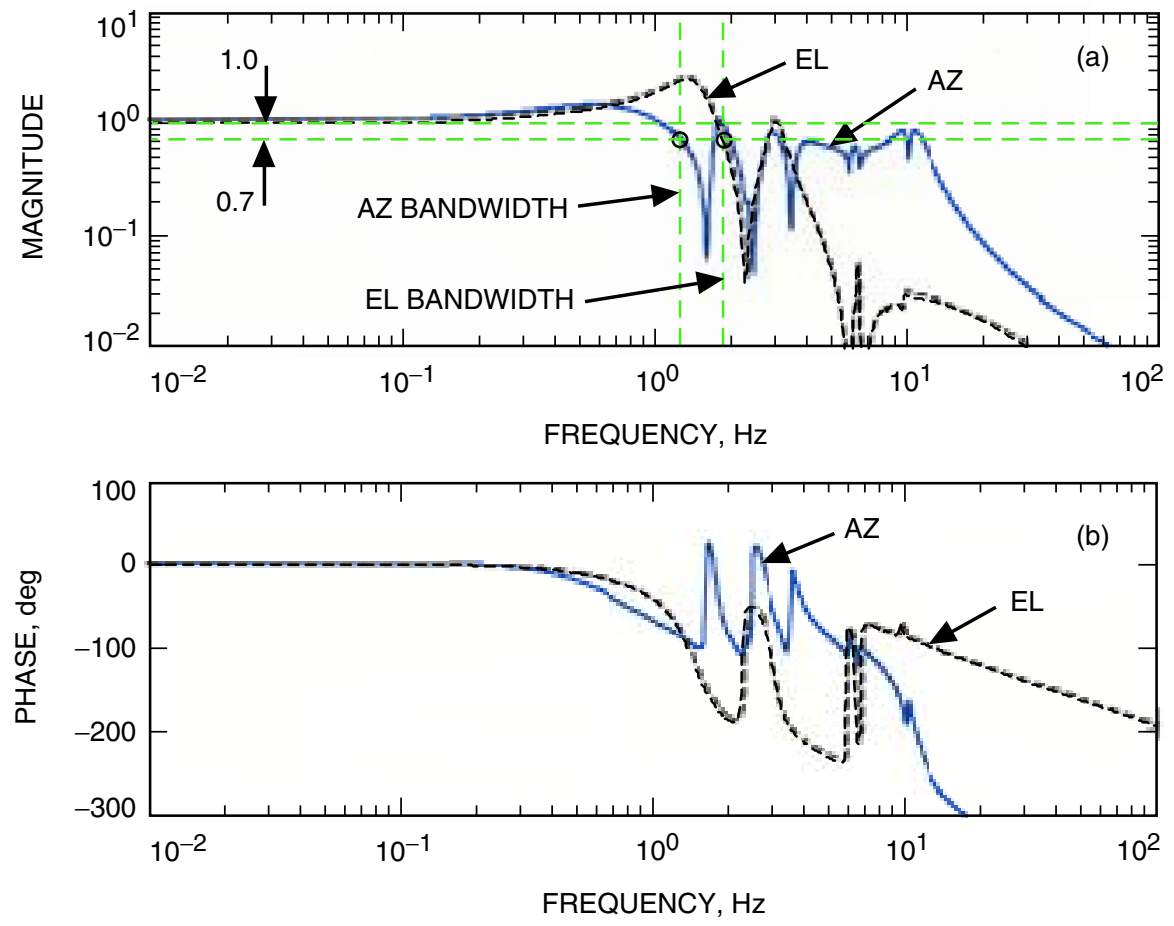

Fig. 8. Position-loop transfer function in azimuth (solid line) and elevation (dashed line): (a) magnitude and (b) phase. 

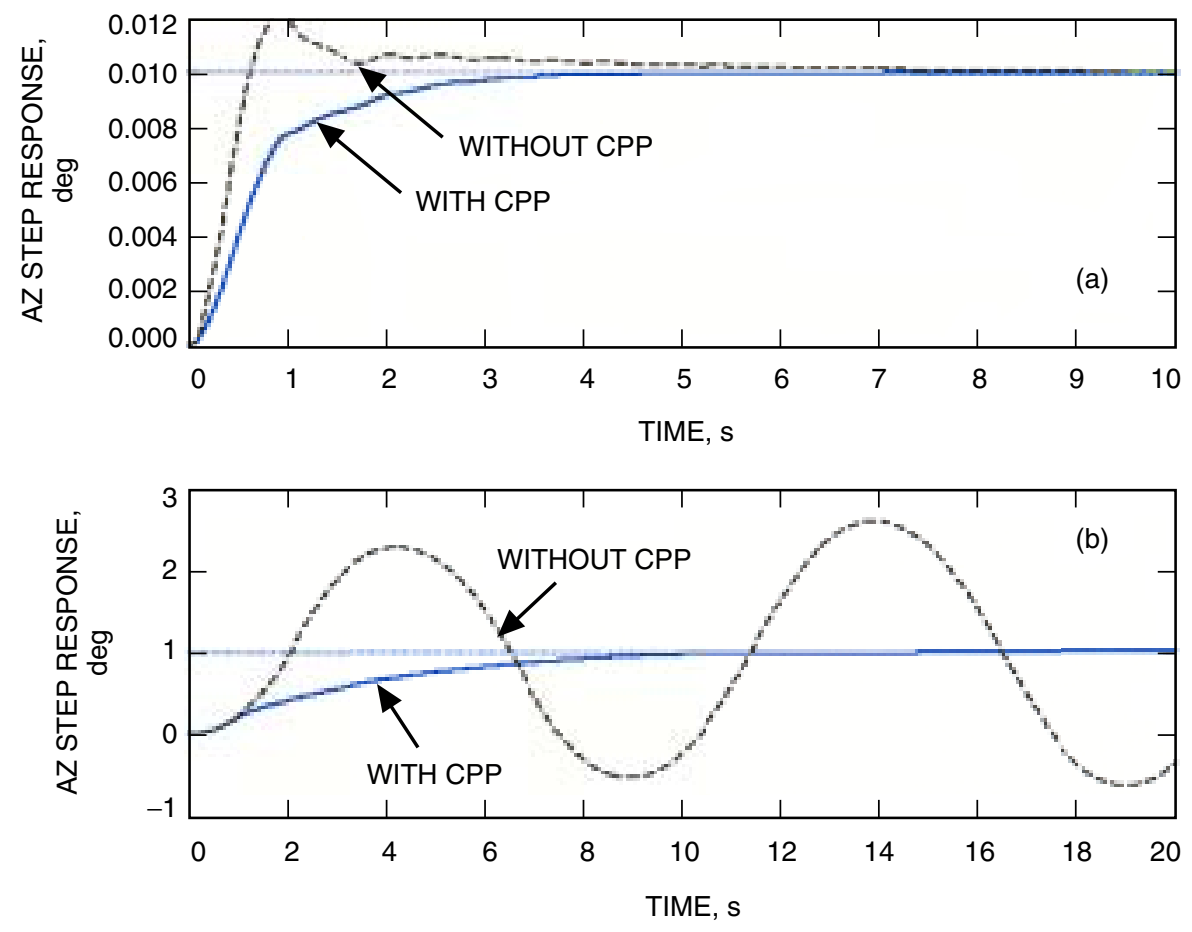

Fig. 9. Telescope responses in azimuth, with the CPP (solid line) and without the CPP (dashed line) to a: (a) 0.01-deg step and (b) 1-deg step.

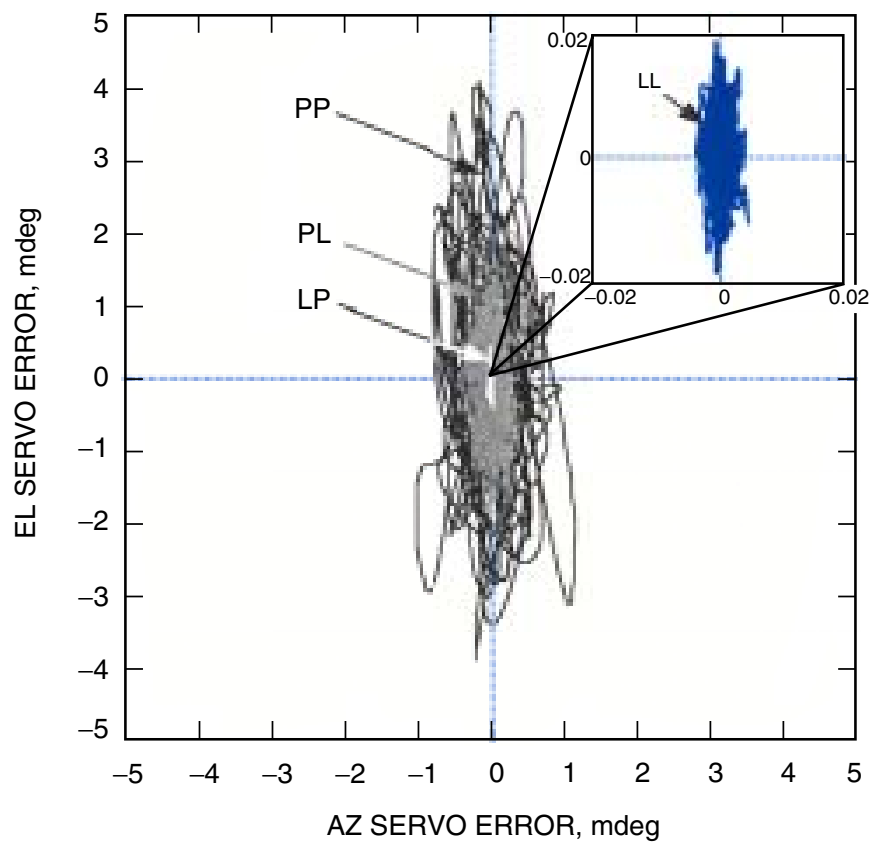

Fig. 10. Telescope servo error in 12-m/s wind gusts: with the PP control system (black), with the PL control system (gray), with the LP control system (white), and with the LL control system (zoomed insert). 
The PP control system analysis showed also that the LMT is a sturdy structure. Its fundamental frequency is $1.7 \mathrm{~Hz}$ (for a typical radio telescope, it will be $1.3 \mathrm{~Hz}$, as shown in Fig. 11). The rigid structure allowed for high gains of PI controllers (3.0 proportional gain and 1.0 integral gain). For comparison, the NASA Deep Space Network antenna fundamental frequency is $1.8 \mathrm{~Hz}$ (although, as a smaller structure, it should have a higher frequency), and allows for 0.5 proportional gain and 0.1 integral gain (3.0 proportional gain and 1.0 integral gain destabilize it). Higher gains mean wider bandwidth (over $1 \mathrm{~Hz}$ ), faster response (a settling time of $3 \mathrm{~s}$ ), and improved wind-disturbance rejection properties. In conclusion, the LMT performs better than could be expected for the PP-type of control system.

\section{The PL Control System}

The PL control system consists of the LQG controller in the position loop and the PI controller in the rate loop.

\section{A. Rate Loop}

In this case, the rate-loop model of the telescope is as shown in Fig. 3(a). It consists of the structure and drive model and the azimuth and elevation rate-loop controllers (PI type). The PI gains of the rate controllers were given in Section II.A.

\section{B. Position Loop}

The position loop is presented in Fig. 3, where the PI controllers are replaced with the LQG controllers as in Fig. 3(c). It consists of the same rate-loop model as in the PP control system, the LQG controller with feedforward loop, the command preprocessor, and rate and acceleration limits. The CPP parameters are as follows: $k_{v}=6, k_{o}=0.6$, and $\beta=20$ for azimuth and elevation.

The LQG controller structure is shown in Fig. 12. The controller includes the estimator, which is an analytical model of the telescope. The estimator is driven by the same input $\left(u_{c}\right)$ as the telescope, but

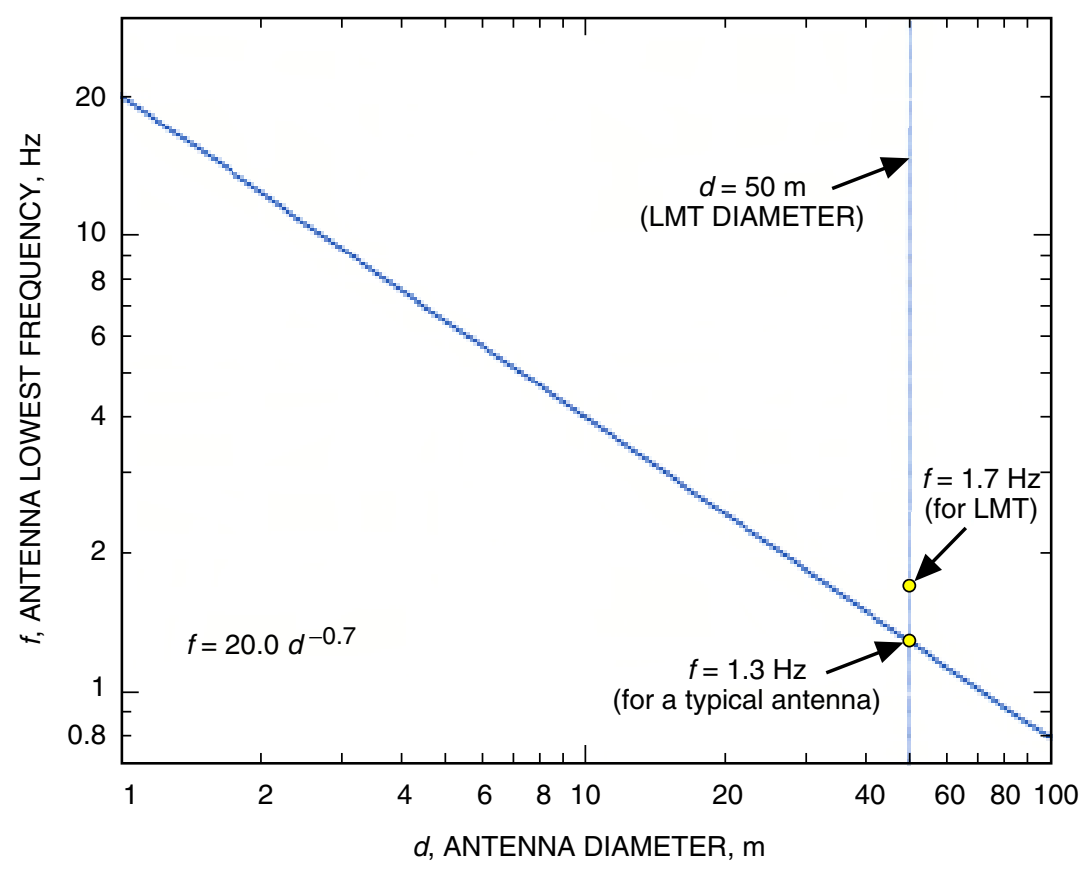

Fig. 11. Antenna fundamental frequency versus antenna diameter. This plot is based on data from the Lowest Servo Resonant Frequency Chart by the Aerospace Corporation. 


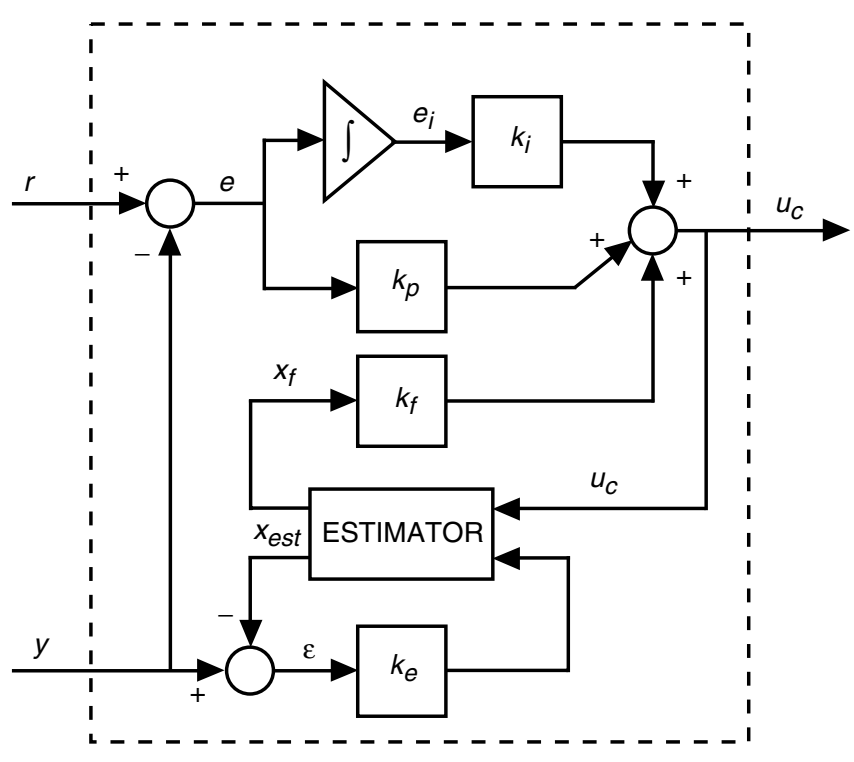

Fig. 12. The LQG controller structure.

also by the estimation error $\varepsilon$ (the difference between the actual rate $y$ and the estimated rate $y_{\text {est }}$ ). The error is amplified with the estimator gain $k_{e}$ to correct for transient dynamics. The estimator outputs are the estimated telescope states that consist of the telescope rate and the telescope flexible deformations $x_{f}$. The latter are the missing vibration measurements, which allow for suppression of the telescope vibrations. The gains of the rate-loop LQG controller were obtained from the LQG design procedure (see [5-7,9]).

We evaluated the performance of the PL control system using settling time, bandwidth, and servo error in wind gusts. The step responses for small (0.01-deg) and large (3-deg) steps are shown in Fig. 13. Figure 13(a) shows 1.6-s settling time in azimuth, 1.2-s settling time in elevation, 18 percent overshoot in azimuth, and 35 percent overshoot in elevation. The position-loop transfer functions for azimuth and elevation are shown in Fig. 14. They show a wide bandwidth of $1.3 \mathrm{~Hz}$ in azimuth and $1.5 \mathrm{~Hz}$ in elevation.

The wind-gust simulations show 0.15-mdeg rms servo error in azimuth and 0.74-mdeg rms servo error in elevation (see the servo error in wind gust plotted in Fig. 10 in gray). These numbers are compared with the PP control system ( 0.35 mdeg in azimuth and $1.4 \mathrm{mdeg}$ in elevation). This means that the LQG controller improves the servo error in wind over the PID controller by a factor of 2.3 in azimuth and a factor of 1.9 in elevation.

The results obtained on the LQG position controller are promising. The telescope had a settling time of $1.6 \mathrm{~s}$, bandwidth of $1.3 \mathrm{~Hz}$, and wind servo error of $0.76 \mathrm{mdeg}$ - or 2 times smaller than that of the PP control system.

\section{The LP Control System}

The LP control system consists of the PID (proportional-integral-derivative) controller in the position loop and the LQG controller in the rate loop. Its Simulink model is shown in Fig. 3(a) and the rate-loop subsystem in Fig. 3(b).

\section{A. Rate Loop}

The Simulink model of the open-loop telescope is as shown in Fig. 3(b), with the rate feedback removed. The open-loop model is scaled to obtain a maximal rate of $1 \mathrm{deg} / \mathrm{s}$ for a $10-\mathrm{V}$ command (a 

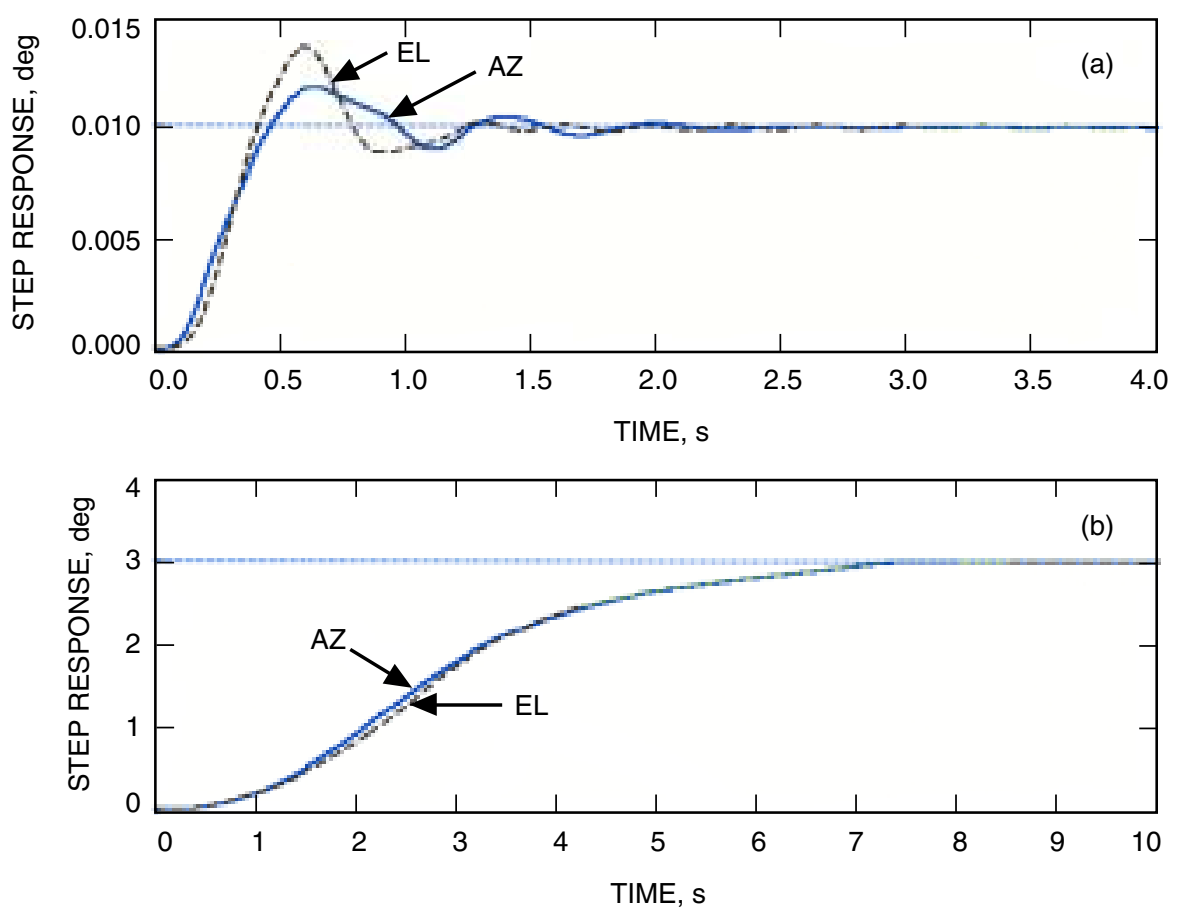

Fig. 13. The PL control system responses to: (a) 0.01-deg step and (b) 3-deg step.
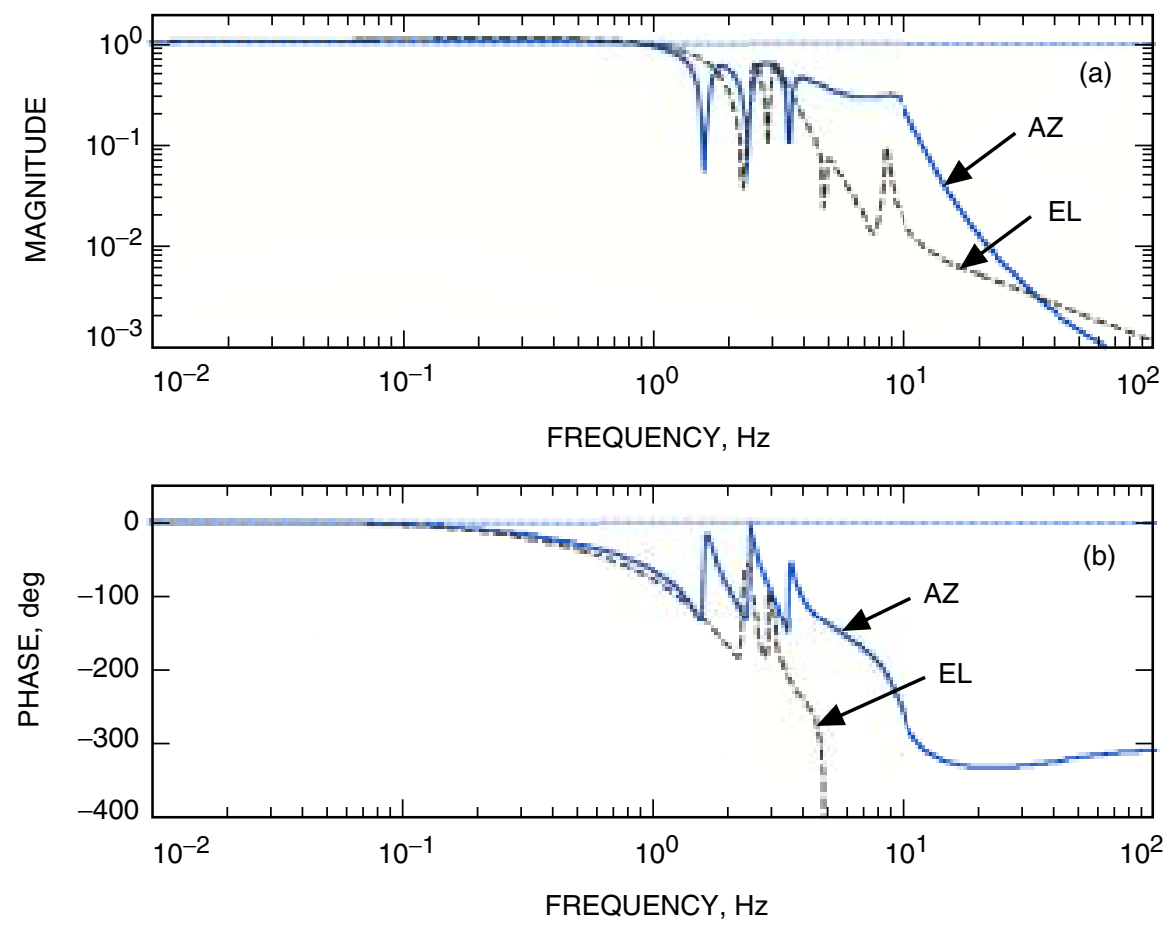

Fig. 14. Azimuth and elevation transfer functions of the PL control system: (a) magnitudes and (b) phases. 
standard input to motor drives). For this open-loop model, we designed an LQG controller and evaluated the performance of the rate-loop LQG controller using its step responses and transfer functions of the azimuth and elevation rate-loop; the settling time is $0.2 \mathrm{~s}$ in azimuth and elevation, and the bandwidth is $1.6 \mathrm{~Hz}$ and $1.8 \mathrm{~Hz}$ in azimuth and elevation, respectively.

\section{B. Position Loop}

The position loop is as shown in Fig. 3, but the azimuth and elevation rate controller are now of the LQG type. Besides the rate loop, the control system consists of the PID controller with a feedforward loop, the command preprocessor, and rate and acceleration limiters. The feedforward loop forwards the command rate to the rate-loop input. The following PID gains were selected: proportional gain, 10; integral gain, 6; and derivative gain, 5, for both azimuth and elevation. The CPP parameters are as follows: $k_{v}=6, k_{o}=0.93$, and $\beta=30$, for azimuth and elevation.

The position-loop performance was evaluated using step responses, bandwidth, steady-state errors due to rate offsets, and servo errors in wind gusts. The step responses for small (0.01-deg) and large (3-deg) steps are shown in Fig. 15, showing 0.6-s settling time and no overshoot for both azimuth and elevation.

The position-loop transfer functions for azimuth and elevation are shown in Fig. 16. They show a wide bandwidth of $200 \mathrm{~Hz}$ in azimuth and $20 \mathrm{~Hz}$ in elevation. The steady-state error due to rate offsets is zero.

The wind-gust simulations for a $12-\mathrm{m} / \mathrm{s}$ wind are plotted in Fig. 10 (white). The figure shows 0.012-mdeg rms servo error in azimuth and 0.150-mdeg rms servo error in elevation. These small numbers show that, as compared with the PP control system, the LQG controller in the rate loop improves the servo error in wind by a factor of 30 in azimuth and a factor of 10 in elevation.

The results obtained on the LQG rate controller are very promising. The telescope performance exceeds the expectation, since its settling time is $0.6 \mathrm{~s}$, the bandwidth is $10 \mathrm{~Hz}$, and wind servo error is 0.15 mdeg - or 10 times smaller than with the PID controller.

\section{The LL Control System}

Finally, we designed the telescope control system with the LQG controller in the rate and position loops. This is also a novel configuration in the antenna industry.

\section{A. Rate Loop}

The rate loop is the same as for the LP control system.

\section{B. Position Loop}

For the given rate loop, the position-loop controller was designed to minimize the servo error in the wind gusts. The position-loop characteristics are plotted in Figs. 17 and 18. From Fig. 17, it follows that the system settling time is $0.5 \mathrm{~s}$, and there is no overshoot in either azimuth or elevation. From Fig. 18, one can find that the bandwidth is $20 \mathrm{~Hz}$ in azimuth and $40 \mathrm{~Hz}$ in elevation. Finally, the wind-gust simulations for a $12-\mathrm{m} / \mathrm{s}$ wind are plotted in the zoomed insert in Fig. 10. The figure shows 0.0012-mdeg rms servo error in azimuth and 0.0057-mdeg rms servo error in elevation, which give a total rms error of $0.0058 \mathrm{mdeg}$. It is 250 times smaller than the error of the PP control system. Thus, the LL control system performance is the best of all the systems presented, although the system is the most complicated and will require careful tuning of both rate- and position-loop LQG controllers in order to obtain the predicted performance. 

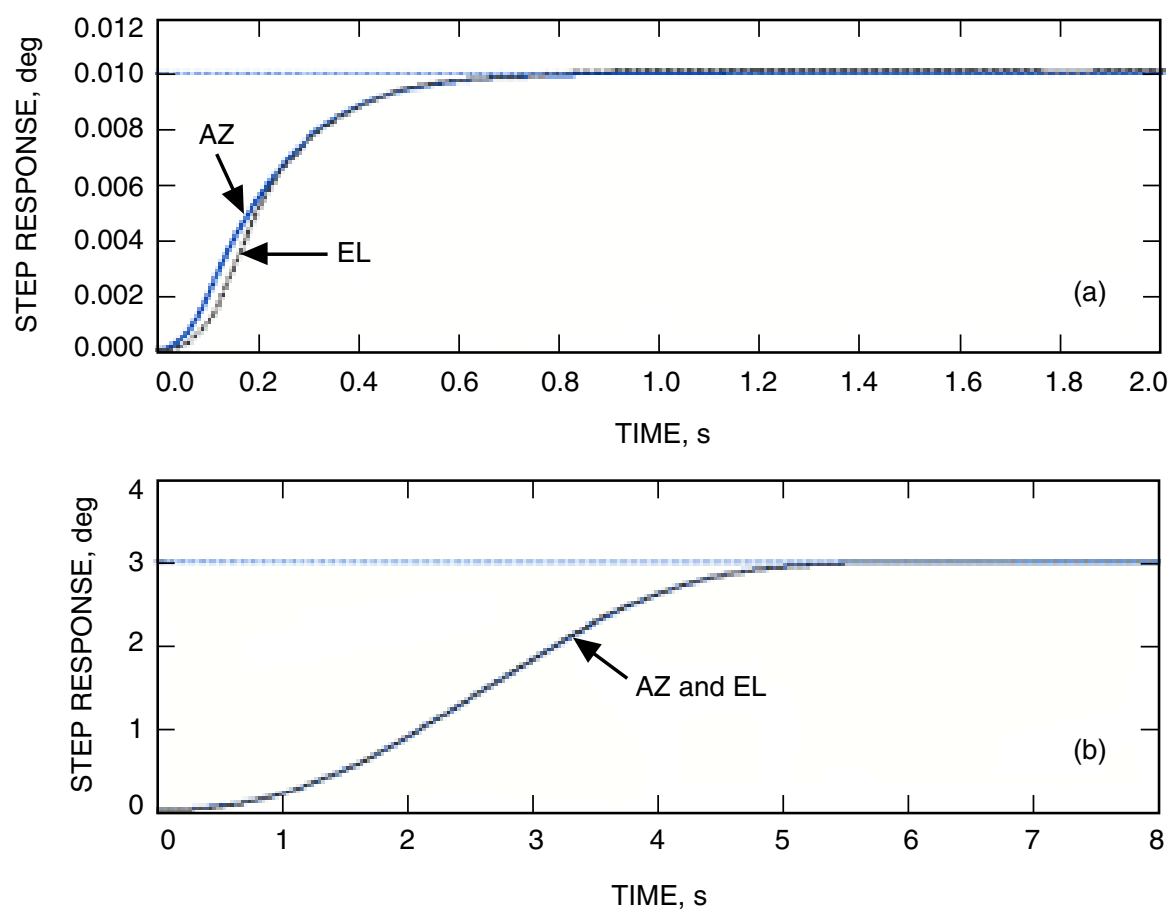

Fig. 15. The LP control system position-loop response to: (a) 0.01-deg step and (b) 3-deg step.
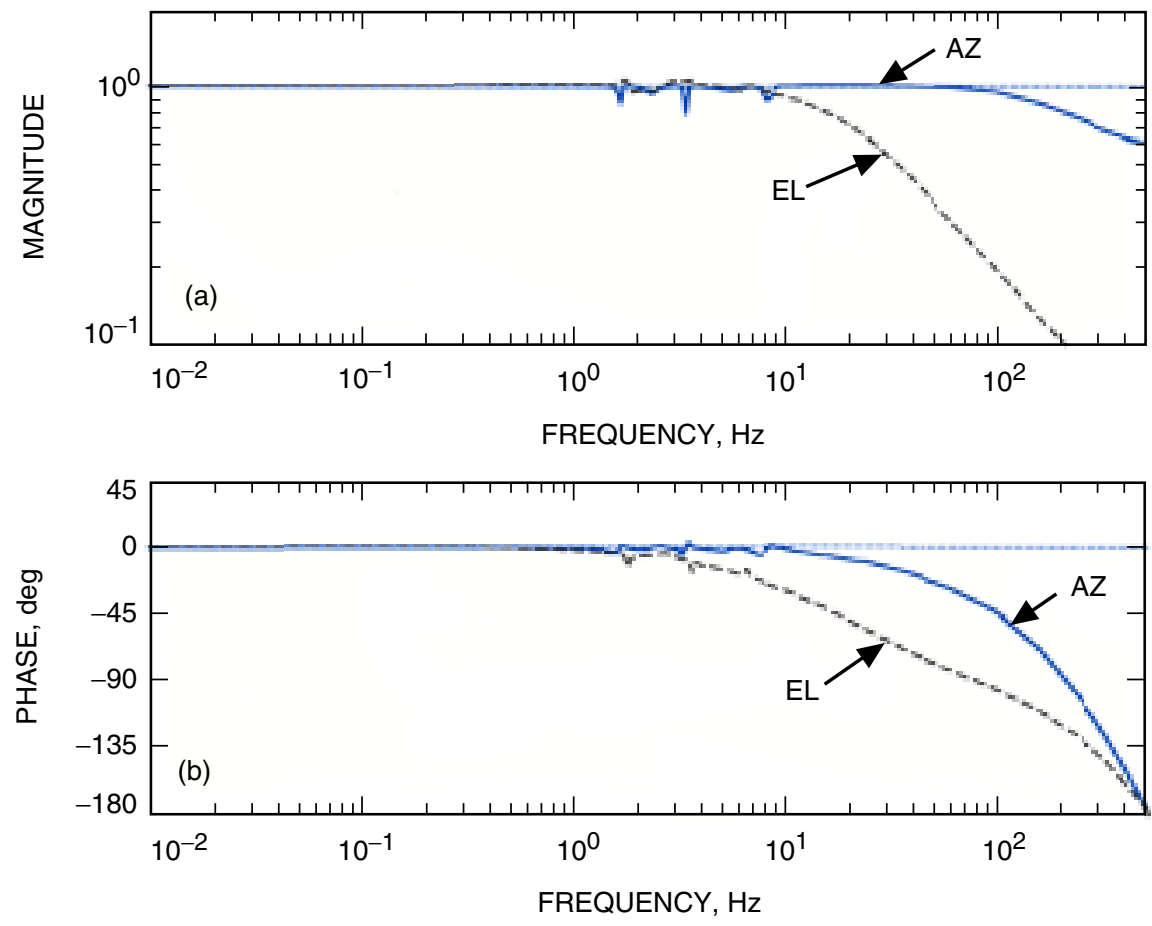

Fig. 16. Azimuth and elevation position-loop transfer functions of the LP control system: (a) magnitudes and (b) phases. 

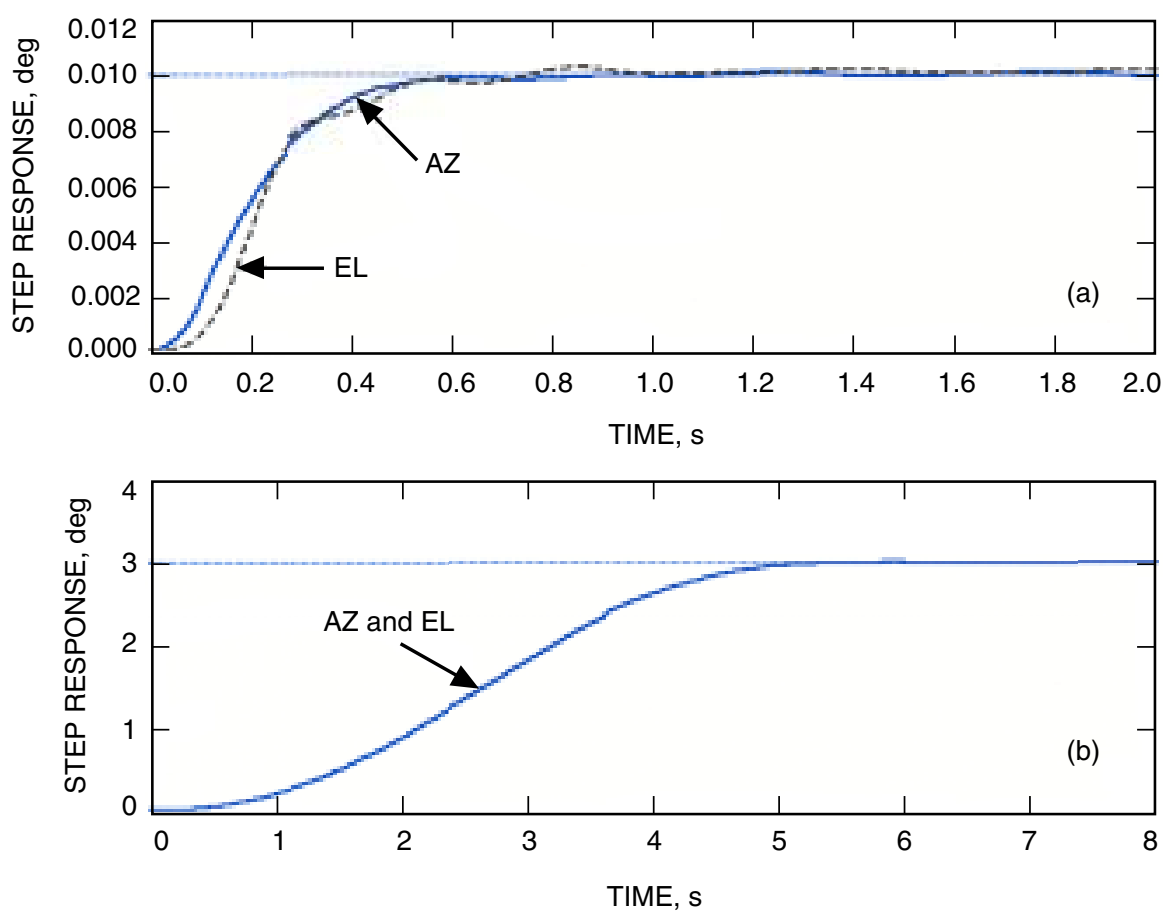

Fig. 17. The LL control system position-loop response to: (a) 0.01-deg step and (b) 3-deg step.
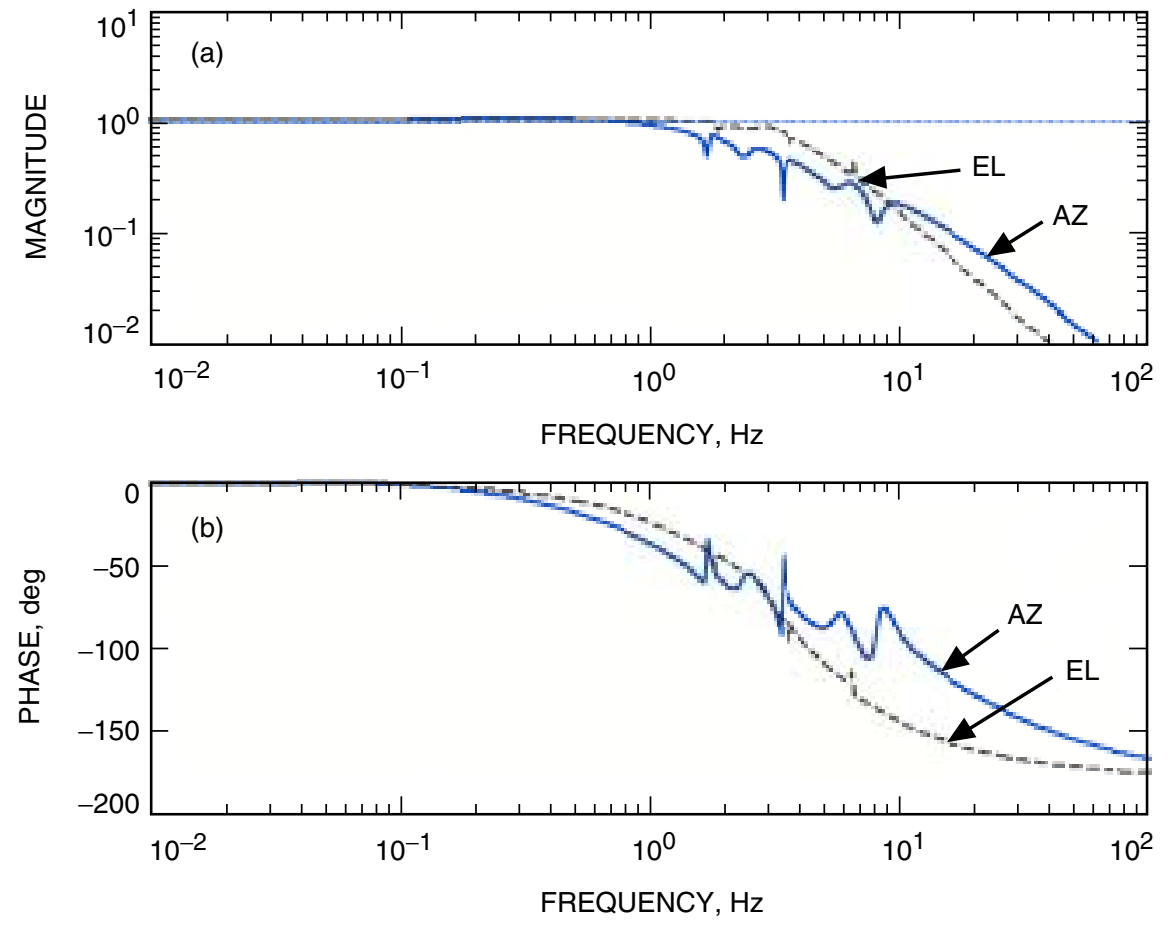

Fig. 18. Azimuth and elevation position-loop transfer functions of the $L L$ control system: (a) magnitudes and (b) phases. 


\section{Conclusions}

This article presented the LMT control systems and evaluated their performances, which are summarized in Table 2.

The PP control system is widely used in the antenna and radio telescope industry. The analysis shows that the LMT structure is exceptionally rigid; thus, the PP control system shows improved pointing accuracy when compared with similar control systems applied to typical antennas or telescopes. The PL control system is implemented at the NASA Deep Space Network antennas, and its pointing precision in wind is twice as good as that of the PP system. The LP control system has not been implemented yet at known antennas or radio telescopes, and the analysis shows that its pointing accuracy in wind is ten times better than that of the PP system. This significant reduction was achieved because of the expanded bandwidth of the rate loop. The LL control system also has not been implemented yet at known antennas or radio telescopes. The analysis shows that its pointing accuracy in wind is 250 times better than that of the PP system. Both the LP and LL control systems are worth further investigation in hope that their implementation will meet the stringent pointing requirements.

Finally, some comments on the obtained performance estimates of the telescope are necessary. The estimates, the best currently available, include some unknown factors. First, the structural model factor: the presented telescope performance is based on the analytical models of the structure and the drives, which do not represent accurate dynamics of the telescope. To improve the accuracy, a model will be derived from the system identification and data collected at the real telescope. Next, the wind disturbance torques are applied to the drives, while in reality the wind acts on the entire structure, including the dish surface. Finally, the RF beam movement is the ultimate goal of the control, and it is not directly measured. Instead, azimuth and elevation encoders are used, which only partially reflect the beam position. The encoders - although relatively precise - cannot exactly measure the actual beam position due to their distant location from the beam focal point, which is the RF beam location.

The performed analysis shows the impact of the location of telescope controllers on the telescope's pointing accuracy and should help to select the most effective system (in terms of cost and precision).

Table 2. Performance of the PP, LP, PL, and LL control systems of the Large Millimeter Telescope.

\begin{tabular}{ccccc}
\hline $\begin{array}{r}\text { Control } \\
\text { system }\end{array}$ & $\begin{array}{c}\text { Settling } \\
\text { time, s }\end{array}$ & $\begin{array}{c}\text { Overshoot, } \\
\text { percent }\end{array}$ & $\begin{array}{c}\text { Bandwidth, } \\
\mathrm{Hz}\end{array}$ & $\begin{array}{c}\text { Wind-gust } \\
\text { servo error, } \\
\text { mdeg }\end{array}$ \\
\hline PP & 3.0 & 20 & 1.2 & 1.48 \\
LP & 0.6 & 0 & 20 & 0.15 \\
PL & 1.4 & 20 & 1.4 & 0.76 \\
LL & 0.5 & 0 & 20 & 0.004 \\
\hline
\end{tabular}




\section{References}

[1] P. Eisentraeger and M. Suess, "Verification of the Active Deformation Compensation System of the LMT/GMT by End-to-End Simulation," Proceedings of the SPIE, Radio Telescopes, vol. 4015, pp. 488-497, 2000.

[2] W. Gawronski, "Antenna Control Systems: From PI to $\mathrm{H}_{\infty}$," IEEE Antennas and Propagation Magazine, vol. 43, no. 1, pp. 52-60, 2001.

[3] W. Gawronski and W. T. Almassy, "Command Preprocessor for Radio Telescopes and Microwave Antennas," IEEE Antennas and Propagation Magazine, vol. 44, no. 2, pp. 30-37, 2002.

[4] W. Gawronski, "Three Models of Wind-Gust Disturbances for the Analysis of Antenna Pointing Accuracy," The Interplanetary Network Progress Report 42-149, January-March 2002, Jet Propulsion Laboratory, Pasadena, California, pp. 1-15, May 15, 2002.

http://ipnpr.jpl.nasa.gov/tmo/progress_report/42-149/149A.pdf

[5] W. Gawronski, C. Racho, and J. Mellstrom, "Application of the LQG and Feedforward Controllers for the DSN Antennas," IEEE Transactions on Control Systems Technology, vol. 3, pp. 417-421, 1995.

[6] W. Gawronski, "Linear Quadratic Controller Design for the Deep Space Network Antennas," AIAA Journal of Guidance, Control, and Dynamics, vol. 17, pp. 655660, 1994.

[7] W. Gawronski, Dynamics and Control of Structures, New York: Springer, 1998.

[8] H. J. Kaercher and J. W. M. Baars, "The Design of the Large Millimeter Telescope/Gran Telescopio Milimetrico (LMT/GTM)," Proceedings of the SPIE, Radio Telescopes, vol. 4015, pp. 155-168, 2000.

[9] E. Maneri and W. Gawronski, "LQG Controller Design Using GUI: Application to Antennas and Radio-Telescopes," ISA Transactions, vol. 39, no. 2, pp. 243$264,2000$. 